\title{
Mean-field approximation for a limit order driven market model
}

\author{
František Slanina \\ Institute of Physics, Academy of Sciences of the Czech Republic, \\ Na Slovance 2, CZ-18221 Praha, Czech Republic \\ e-mail: slanina@fzu.cz
}

\begin{abstract}
The mean-field variant of the model of limit order driven market introduced recently by Maslov is formulated and solved. The agents do not have any strategies and the memory of the system is kept within the order book. We show that he evolution of the order book is governed by a matrix multiplicative process. The resulting stationary distribution of step-to-step price changes is calculated. It exhibits a power-law tail with exponent 2 . We obtain also the price autocorrelation function, which agrees qualitatively with the experimentally observed negative autocorrelation for short times.
\end{abstract}

PACS numbers: 05.40.-a; 89.90.+n

\section{INTRODUCTION}

The complexity of market behavior, seen as a particular example of a natural phenomenon, fascinated physicists since long time ago [1]. The main source of interest comes from a kind of critical behavior, made explicit by the power-law distribution and scaling in the economic time series, first observed by Mandelbrot (see [2] and references therein) and studied in detail by Mantegna and Stanley [3 5] and subsequently by many others (see e. g. 6.11).

The scaling and multifractal properties call for an explanation in terms of a model mimicking the behavior of individual agents in the market. The physicist's optimism in looking for such a model might be strengthened by recent successes in modeling other social phenomena, e. g. in the cellular-automata models of traffic [12]. The idea consists in assuming that all the overwhelming complexity of a human being is irrelevant in certain special conditions: when driving a car, only a very basic set of behaviors is at work. Similarly, it is assumed that a trading agent, when put on the floor, follows only a limited set of instincts or acquired patterns. Therefore, in this approach the economic complexity is not due to the intrinsic complexity of each agent (as usual hand-waving arguments by liberal opponents of "reductionism" state), but an emergent property of a large set of non-linearly interacting simple units.

Many microscopic stock market models emerged during last several years. One of the first ones was the model introduced by Levy, Levy and Solomon 13 15 which captures essential features of the price fluctuations and explains also the power law distribution of investor's wealth, which is the famous Pareto law.

Another approach was used in the model of Bak, Paczuski and Shubik [16]: buyers and sellers are represented by particles subject to a reaction-diffusion process. Introduction of a non-trivial strategy of the agents lead to a realistic value of the Hurst exponent for the price fluctuations. The simpler version of the model was then solved analytically [17]. Variety of other approaches were investigated 18 34.

The above mentioned models mostly do not take into account the realistic details of the price formation through the book of orders. This mechanism was implemented in the model set up by Maslov [35] and a similar perspective was then used in a recent series of papers by Matassini and Franci 36 38. The book of orders perspective to market modeling was empirically investigated in 39.40 .

With such a diversity of models, most of which giving plausible explanation of observed facts a question arises, whether there is a common mechanism behind various approaches, making them essentially equivalent. Indeed, it was found that such a mechanism may be the multiplicative stochastic process repelled from zero, or multiplicative-additive process. It was studied thoroughly by various authors and in diverse contexts [15, 41, 52]. The goal we pose in this work is to show that essentially the same mechanism is responsible for the power-law distribution of price changes also in the limit-order model.

\section{LIMIT ORDER DRIVEN MARKET MODEL}

Recently Maslov [35,39] proposed a model, based on the assumption that there are two kinds of market participants. Prudent investors place their orders at prescribed price and a trade occurs as soon as there is anybody accepting that price. On the other hand, speculators buy and sell at any moment at the price which is available in the market. The price signal $p(t)$ was found to have power-law spectrum, with Hurst exponent $H=1 / 4$. The price changes during unit time interval $x=p(t+1)-p(t)$ have probability distribution which follow clear power law $P(x) \sim x^{-(1+\alpha)}$ in two regimes. For small $x$ the exponent is $1+\alpha_{1}=0.6 \pm 0.1$, while in the regime of large price changes the exponent is $1+\alpha_{2}=3 \pm 0.2$. These values are to be compared to the experimentally found $1+\alpha_{1} \simeq 2.5$ and $1+\alpha_{2} \simeq 4$ respectively 6.9 .11.

The model [35] can be described as follows. There are 
orders to buy and sell placed on a straight line, which is the axis of the price $x$. In stable situation, all buy orders are lower than all sell orders, so that we can describe the state by single function $\rho(x)$, density of the orders, and a number $\xi$ which is the last realized price. Then, all $x<\xi$ correspond to buy, all $x>\xi$ to sell orders.

Two events can change the state. First, new limit orders may be dropped, such that $\rho(x) \rightarrow \rho(x)+\eta(x-\xi)$ We suppose the function $\eta(x)$ equal in all events and symmetric, $\eta(x)=\eta(-x)$. Second, a market-price order can arrive. An order to buy an amount $s$ results to clearing all sell orders up to the price $\xi+x_{+}$, where

$$
\int_{\xi}^{\xi+x_{+}} \rho(x) \mathrm{d} x=s
$$

The new price is then $\xi \rightarrow \xi+x_{+}$, so that $x_{+}$is the price increment, while the new density is $\rho(x) \rightarrow(1-\theta(x-$ $\left.\xi) \theta\left(\xi+x_{+}-x\right)\right) \rho(x)$. Analogical formulae will hold for the sell order.

As we can see, there are no strategies which would lead the agents to perform specific actions. The model is barely stochastic. The long-term memory of the system and thus a possible power-law behavior stems from the order book, or the time-dependent density function $\rho(x)$ which may keep arbitrarily old orders.

\section{MATRIX FORMULATION}

Our essential approximation to this model will consist in supposing uniform density of orders on each side from the current price level $\xi$. In reality, both dropping new limit orders and clearing them by market orders makes the density of states uneven and fluctuating. When supposing that after an event the uniform density of states is restored, we make a kind of "mean-field" approximation: the actual position of each limit order is not important, as if they were freely moving particles making an effective medium, within which the price fluctuates. High density of the medium will result in smaller price fluctuations and vice versa.

The density on the upper side will be denoted $\rho_{+}$, on the lower side $\rho_{-}$. It is convenient to describe the densities in terms of the potential price changes, which would occur if a market-price order arrives. They are simply $x_{+}=s / \rho_{+}$for buy and $x_{-}=s / \rho_{-}$for sell order. The numbers $x_{ \pm}$form a vector $X=\left(\begin{array}{c}x_{+} \\ x_{-}\end{array}\right)$which performs a stochastic process, as the densities $\rho_{ \pm}$and therefore the numbers $x_{ \pm}$are updated after arrival of each order. The dynamical rules of the process represent a simplified version of the limit order driven dynamics.

There are be three types of events: (i) dropping of limit orders (ii) market-price order to buy, and (iii) marketprice order to sell. We suppose that all market price orders have the same volume $s$ and all limit-order events the same volume $v$. Further we assume that market orders to buy and sell occur with the same probability. In order to keep the total number of limit orders constant on average, we should suppose that at given moment there is probability $p=s /(s+v)$ to drop a limit orders. Each of the market-price events (ii) and (iii) have then equal probability $(1-p) / 2$. (Here we tacitly assume that the limit orders which are not met last forever. One can also investigate more realistic variants, where the limit orders slowly die out).

Let us investigate first the consequence of an arrival of a market-price order to buy. By definition, the price level increases by $x_{+}$. As the density of orders is constant, average density on the right-hand side from the new price is unchanged. If now another buy order arrives, it finds the same density and the price change is the same too. Therefore, the new value of $x_{+}$is equal to the old one, $x_{+} \rightarrow x_{+}$. On the other hand, if now a new market order to sell arrives, there are no limit orders in the interval of width $x_{+}$below the current price level, and when we go further down, there is a constant density $s / x_{-}$. As a result, the price decreases by $x_{+}+x_{-}$. Hence, the new value of $x_{-}$is $x_{-} \rightarrow x_{+}+x_{-}$.

To sum it up, the effect of the buy order consists in the replacement

$$
\begin{aligned}
& x_{+} \rightarrow x_{+} \\
& x_{-} \rightarrow x_{+}+x_{-} .
\end{aligned}
$$

It can be expressed in matrix form

$$
X \rightarrow X^{\prime}=T_{+} X
$$

where

$$
T_{+}=\left(\begin{array}{ll}
1 & 0 \\
1 & 1
\end{array}\right) \text {. }
$$

Similarly, for the action of a sell order we get

$$
X \rightarrow X^{\prime}=T_{-} X
$$

where

$$
T_{-}=\left(\begin{array}{ll}
1 & 1 \\
0 & 1
\end{array}\right) .
$$

Now we turn to the changes due to dropping limit orders. It is necessary to specify the function $\eta(x)$, representing the average volume of orders set at distance $x$ from the current price. As we already mentioned, we suppose it to be an even function. Moreover, the volume was supposed to be fixed, $\int \eta(x) \mathrm{d} x=v$. We apply here the simplest choice $\eta(x)=\frac{v}{2}(\delta(x-d)+\delta(x+d))$, which means that all new orders are placed at the same distance $d$ from the current price, either below (buy) or above (sell). This distribution reflects the fact, that the limit orders are not typically set arbitrarily close to the current price, but there is a certain minimum offset $d$. 
Dropping limit orders affects the vector $X$ according to the formula

$$
x_{ \pm} \rightarrow \frac{1}{2}(3-1 / p) x_{ \pm} .
$$

Indeed, a buy (or sell) order will annihilate the amount $v / 2$ from the just deposited limit order and amount $s-$ $v / 2$ from the original density of old limit orders. The shift is therefore $x_{ \pm}=(s-v / 2) / \rho_{ \pm}$. Writing $v$ in terms of the probability $p$, i. e. $v=(1 / p-1) s$, we obtain the formula (7). So, in matrix form we have

$$
X \rightarrow X^{\prime}=S X
$$

where

$$
S=\frac{1}{2}\left(3-\frac{1}{p}\right)\left(\begin{array}{ll}
1 & 0 \\
0 & 1
\end{array}\right) .
$$

The price changes only after a market order is issued, while dropping limit orders leaves the price unchanged. So, between two subsequent shifts of the price, $m \geq 0$ limit orders can arrive, with probability $P_{m}(m)=$ $(1-p) p^{m}$. The change of the vector $X$ due to one market order and $m$ limit orders is $X \rightarrow S^{m} T_{ \pm} X$. When calculating the evolution of the probability distribution for $X$, we should sum over all possible realizations. Hence, the probability distribution for the vector $X$ should satisfy the equation

$$
\begin{aligned}
& P_{X}(X)= \\
& \frac{1}{2} \sum_{\sigma= \pm} \sum_{m=0}^{\infty} \int \mathrm{d} X^{\prime} P_{m}(m) P_{X}\left(X^{\prime}\right) \delta\left(X-S^{m} T_{\sigma} X^{\prime}\right)
\end{aligned}
$$

in the stationary state.

\section{DISTRIBUTION OF PRICE CHANGES}

We will make a further approximation at this stage. The matrix $S$ is simply a unit matrix multiplied by a constant. If the same were true also for the matrices $T_{ \pm}$, the process would be reduced to a simple multiplicative random walk, whose properties are well known and their relevance in modeling price fluctuations is testified by a series of models, as mentioned in the Introduction.

Our approximation will consist first in replacing the matrices $T_{ \pm}$by the average $\bar{T}=\frac{1}{2}\left(T_{+}+T_{-}\right)$and furthermore, we will take only the highest eigenvalue of the matrix $\bar{T}$, which is $3 / 2$. Then, instead of a pair of price changes $x_{+}$and $x_{-}$we have a single scalar quantity $x$, describing the absolute value of the price change.

Note that the same results can be obtained by assuming from beginning, that $x_{+}=x_{-}$, i.e. that the density of states is equal on both sides of the price level. This means, that we make a further "mean-field" approximation, suppressing not only the fluctuations along the price axis, but also fluctuations from one side to the other of the already averaged density of states.

This way we define our multiplicative random process. The fact that there is a small but finite offset $d$ in placing the limit orders ensures that the values of $x_{ \pm}$(therefore also of $x$ ) cannot be smaller than $d$. This feature plays the role of "repulsion from zero", which was found essential for establishing the power-law tails 42, 45. and is usually guaranteed by the additive term 43,46 .

For the probability distribution of the price changes we obtain

$$
\begin{aligned}
P(x) & =\sum_{m=0}^{\infty}(1-p) p^{m} \times \\
& \times \int \mathrm{d} x^{\prime} P\left(x^{\prime}\right) \delta\left(x-\frac{3}{2} x^{\prime}\left(\frac{3-\frac{1}{p}}{2}\right)^{m}\right)
\end{aligned}
$$

and assuming a power-law tail of the probability distribution in the form $P(x) \sim x^{-1-\alpha}$ we obtain the following equation for the exponent

$$
\sum_{m=0}^{\infty}(1-p) p^{m}\left(\frac{3}{2}\left(\frac{3-\frac{1}{p}}{2}\right)^{m}\right)^{\alpha}=1 .
$$

As can be easily checked, apart from the trivial solution $\alpha=0$ it has a non-trivial solution $\alpha=1$, independent of $p$. Therefore, the distribution of price changes has a power-law tail

$$
P(x) \simeq x^{-2} .
$$

Note that the calculation could be further simplified by writing the equation analogical to (11), relating the probability distribution just after single step. Then, instead of the sequence of steps consisting of one market order followed by $m$ limit orders we have one step being either limit or market order. This equation gives precisely the same power-law tail. However, such an approach is slightly inconsistent, because setting a limit order does not imply any trade, thus the price change at this moment is zero.

\section{PRICE AUTOCORRELATION FUNCTION}

One of the well-known facts about financial data series is the negative short-time autocorrelation of price changes [6]. Here we will show, how this effect naturally emerges from the matrix nature of our stochastic process.

We will compute the autocorrelation function defined as

$$
C(t, t+\tau)=\frac{\langle x(t) x(t+\tau)\rangle}{\sqrt{\left\langle x^{2}(t)\right\rangle\left\langle x^{2}(t+\tau)\right\rangle}}
$$

where $x(t)$ is the actual price change at time $t$ and $\tau \geq 1$. We will denote $M(t) \in\left\{S, T_{+}, T_{-}\right\}$the matrix describing the action performed at time $t$ and $P_{M}(M)$ its probability distribution. Of course, we introduced already $P_{M}\left(T_{-}\right)=P_{M}\left(T_{+}\right)=(1-p) / 2$ and $P_{M}(S)=p$. 
Now we introduce the function of taking the price change from the vector $X=\left(\begin{array}{l}x_{+} \\ x_{-}\end{array}\right)$

$$
\begin{aligned}
\mathcal{X}(X ; M) & =x_{+} \quad \text { if } M=T_{+} \\
& =0 \quad \text { if } M=S \\
& =x_{-} \text {if } M=T_{-} .
\end{aligned}
$$

Note that the operator $\mathcal{X}$ is linear in the argument $X$. Then

$$
\langle x(t) x(t+\tau)\rangle=\int \mathrm{d} X P_{X}(X) \widetilde{C}(X)
$$

where

$$
\begin{aligned}
& \widetilde{C}(X)=\sum_{M(t)} \ldots \sum_{M(t+\tau)} \prod_{i=0}^{\tau} P_{M}(M(t+i)) \times \\
& \times \mathcal{X}(X ; M(t)) \mathcal{X}(M(t+\tau-1) \ldots M(t) X ; M(t+\tau))
\end{aligned}
$$

We find easily, using the linearity if the operator $\mathcal{X}$ and introducing the sign vector $E=(1,-1)$

$$
\begin{aligned}
\widetilde{C}(X)=\frac{1-p}{2} E & \left(\sum_{M} P_{M}(M) M\right)^{\tau-1} \times \\
& \times\left(x_{+} T_{+}-x_{-} T_{-}\right) X .
\end{aligned}
$$

The multiplication by $E$ extracts only the lower one of the two eigenvalues of the averaged matrix

$$
\sum_{M} P_{M}(M) M=\frac{1}{2}\left(\begin{array}{ll}
1+p & 1-p \\
1-p & 1+p
\end{array}\right) .
$$

The lower eigenvalue is $p$, hence

$$
\widetilde{C}(X)=-(1-p) p^{\tau-1} x_{+} x_{-} .
$$

We can calculate similarly the corresponding expression for the denominator of the equation (15). We obtain at the end

$$
C(t, t+\tau)=-p^{\tau-1} \frac{2\left\langle x_{+} x_{-}\right\rangle}{\left\langle x_{+}^{2}\right\rangle+\left\langle x_{-}^{2}\right\rangle} .
$$

We can clearly observe the negative autocorrelation which decays with characteristic time which depends on the relative frequency of putting the limit and market orders, measured by the probability $p$. The result obtained suffer from the divergence of second moments of the variables $x_{+}, x_{-}$, resulting from the power-law tail calculated in the last section. However, only the ratio of the moments enter the formula (21). Moreover, if we suppose as an initial condition a distribution for $x_{+}, x_{-}$ with finite moments, the moments will remain finite for any finite time and we naturally expect that the ratio of the second moments will converge to a finite value even if the second moments themselves diverge.

\section{CONCLUSIONS}

In conclusion, we solved in the mean-field approximation the Maslov model of stock market fluctuations. We found a stationary distribution of price changes with a power law tail with the exponent $1+\alpha=2$, which is within the Lévy stable region. We found negative shorttime autocorrelation of the price changes, decaying exponentially with time. The relaxation time depends on the relative frequency of putting market orders and limit orders: the decay is slower if a market order comes only after more limit orders. This is intuitively clear, because it is the market order that ensures the liquidity.

Our result differs in two important points from the simulations of Maslov [35]. First, the numerical value of the power-law tail exponent is different. This can be attributed to the approximation we made in the form of the density of orders $\rho(x)$. Indeed, we assumed, as a zero approximation, constant density. On the other hand, it is known from the solution of the reaction-diffusion model of market [17] that the density may have complicated non-trivial form. Another source of the difference may be the neglect of fluctuations.

Another difference consist in lacking the second powerlaw regime for small price changes. However, as discussed in [35], this second and different power law comes from the fact, that the new limit orders may be placed farther than the reach of the price change. As we implicitly supposed that the new orders are put to very small distance $d$ from the current price, we can observe only the distribution for price changes larger than $d$.

\section{ACKNOWLEDGMENTS}

I am indebted to Y.-C. Zhang and the University of Fribourg, Switzerland, for financial support and kind hospitality. I wish to thank Sergei Maslov for stimulating discussions and many claryfying remarks. This work was supported by the Grant Agency of the Czech Republic, grant project No. 202/01/1091.

[1] P. W. Anderson, K. J. Arrow, and D. Pines (eds.), The Economy as an Evolving Complex System, (Addison Wesley, 1988).

[2] B. B. Mandelbrot, Physica A 263, 477 (1999).

[3] R. N. Mantegna, Physica A 179, 232 (1991).

[4] R. N. Mantegna and H. E. Stanley, Nature 376, 46 (1995).

[5] R. N. Mantegna and H. E. Stanley, Introduction to Econophysics: Correlations and Complexity in Finance, (Cambridge University Press, Cambridge, 1999). 
[6] J.-P. Bouchaud and M. Potters, Theory of Financial Risks, (Cambridge University Press, Cambridge, 2000).

[7] S. Galluccio, G. Caldarelli, M. Marsili, and Y.-C. Zhang, Physica A 245, 423 (1997).

[8] N. Vandewalle and M. Ausloos, Eur. Phys. J. B 4, 257 (1998).

[9] P. Gopikrishnan, M. Meyer, L. A. N. Amaral, and H. E. Stanley, Eur. Phys. J. B 3, 139 (1998).

[10] V. Plerou, P. Gopikrishnan, L. A. N. Amaral, M. Meyer, and H. E. Stanley, Phys. Rev. E 60, 6519 (1999).

[11] P. Gopikrishnan, V. Plerou, L. A. N. Amaral, M. Meyer, and H. E. Stanley, Phys. Rev. E 60, 5305 (1999).

[12] K. Nagel and M. Schreckenberg, J. Phys. I France 2, 2221 (1992).

[13] M. Levy, H. Levy, and S. Solomon, Economics Letters 45, 103 (1994).

[14] M. Levy, H. Levy, and S. Solomon, J. Phys. I France 5, 1087 (1995).

[15] M. Levy and S. Solomon, Int. J. Mod. Phys. C 7, 65 (1996).

[16] P. Bak, M. Paczuski, and M. Shubik, Physica A 246, 430 (1997).

[17] L.-H. Tang and G.-S. Tian, Physica A 264, 543 (1999).

[18] H. Takayasu, H. Miura, T. Hirabayashi, and K. Hamada, Physica A 184, 127 (1992).

[19] A.-H. Sato and H. Takayasu, Physica A 250, 231 (1998).

[20] T. Lux and M. Marchesi, Nature 397, 498 (1999).

[21] R. Cont and J.-P. Bouchaud, Macroeconomic Dynamics 4, 170 (2000).

[22] G. Caldarelli, M. Marsili, and Y.-C. Zhang, Europhys. Lett. 40, 479 (1997).

[23] D. Chowdhury and D. Stauffer, Eur. Phys. J. B 8, 477 (1999).

[24] D. Sornette, D. Stauffer, and H. Takayasu, condmat/9909439.

[25] J.-P. Bouchaud and R. Cont, Eur. Phys. J. B 6, 543 (1998).

[26] G. Iori, adap-org/9905005, to appear in Journal of Economic Behaviour and Organization.

[27] G. Iori, Int. J. Theor. Appl. Finance 3, 467 (2000).

[28] F. Slanina and Y.-C. Zhang, Physica A 272, 257 (1999).

[29] F. Slanina and Y.-C. Zhang, Physica A 289, 290 (2001).

[30] L. Kullmann and J. Kertész, cond-mat/0105473, to appear in Physica A (proceedings of NATO ARW, Prague, 2001).

[31] D. Challet and Y.-C. Zhang, Physica A 246, 407 (1997).

[32] D. Challet, M. Marsili, and Y.-C. Zhang, Physica A 276, 284 (2000).

[33] N. F. Johnson, M. Hart, P. M. Hui, and D. Zheng, Int. J. Theor. Appl. Finance 3, 443 (2000).

[34] D. Challet, M. Marsili, and Y.-C. Zhang, condmat/0101326.

[35] S. Maslov, Physica A 278, 571 (2000).

[36] F. Franci and L. Matassini, cond-mat/0008466.

[37] L. Matassini and F. Franci, cond-mat/0103106.

[38] L. Matassini and F. Franci, Physica A 289, 526 (2001).

[39] S. Maslov and M. Mills, cond-mat/0102518, to appear in Physica A (proceedings of NATO ARW, Prague, 2001).

[40] D. Challet and R. Stinchcombe, cond-mat/0106114.

[41] J. M. Deutsch, Physica A 208, 433 (1994).
[42] M. Levy and S. Solomon, Int. J. Mod. Phys. C 7, 595 (1996).

[43] H. Takayasu, A.-H. Sato, and M. Takayasu, Phys. Rev. Lett. 79, 966 (1997).

[44] D. Sornette, Physica A 250, 295 (1998).

[45] D. Sornette and R. Cont, J. Phys I France 7, 431 (1997).

[46] D. Sornette, Phys. Rev. E 57, 4811 (1998).

[47] O. Biham, O. Malcai, M. Levy, and S. Solomon, Phys. Rev. E 58, 1352 (1998).

[48] M. Marsili, S. Maslov, and Y.-C. Zhang, Physica A 253, 403 (1998).

[49] S. Solomon, in: Application of Simulation to Social Sciences ed. G. Ballot and G. Weisbuch, (Hermes Science Publications, 2000).

[50] Z.-F. Huang and S. Solomon, Eur. Phys. J. B 20, 601 (2001).

[51] S. Solomon and M. Levy, cond-mat/0005416.

[52] J.-P. Bouchaud and M. Mézard, Physica A 282, 536 (2000). 